\title{
Evolução Tardia de Pacientes com Infarto Agudo do Miocárdio Tratados com Stents Farmacológicos na Prática Diária do Mundo Real - Subanálise do Registro DESIRE (Drug-Eluting Stent In the REal World)
}

\author{
Ricardo A. Costa ${ }^{1}$, Amanda G. M. R. Sousa ${ }^{1}$, J. Ribamar Costa Jr. ${ }^{1}$, Adriana Moreira ${ }^{1}$, Galo Maldonado ${ }^{1}$, \\ Manuel N. Cano1', Fausto Feres', César Jardim¹, leda M. Liguori', Marco A. Barbosa', \\ Edson R. Romano' ${ }^{1}$ J. Eduardo Sousa'
}

\section{RESUMO}

Introdução: Estudos prévios comparando stents farmacológicos (SFs) e stents não-farmacológicos sugerem eficácia e segurança dos SFs no tratamento de pacientes com infarto agudo do miocárdio (IAM). No entanto, a evolução tardia de pacientes com IAM tratados com SFs na prática diária permanece desconhecida. Este estudo teve como objetivo investigar o seguimento tardio de pacientes com IAM recente tratados com SFs na prática diária do mundo real. Métodos: Entre maio de 2002 e junho de 2009, 3.018 pacientes não-selecionados com indicação de intervenção percutânea eletiva ou de emergência foram consecutivamente tratados com SFs em uma instituição clínica. O seguimento tardio de até sete anos foi realizado em 98\%, com mediana de 3,4 anos. Os pacientes foram divididos em dois grupos: pacientes com IAM recente $(<30$ dias) e pacientes sem IAM recente. Resultados: Os pacientes com IAM recente tinham menos comorbidades e mais doença multiarterial $(68,1 \%$ vs. $60,7 \%$; $P<0,001)$, lesões com trombo $(13,6 \%$ vs. $1,3 \% ; \mathrm{P}<0,001)$, fluxo TIMI $0 / 1(8,3 \%$ vs. $1,1 \% ; \mathrm{P}<0,001)$ e disfunção moderada/grave do ventrículo esquerdo $(23,2 \%$ vs. $10,9 \% ; \mathrm{P}<0,001)$, comparativamente aos pacientes sem IAM recente. O grupo IAM recente recebeu 1,6 $\pm 0,8$ stent/ paciente e mais inibidores da glicoproteína Ilb/Illa (19,6\% vs. $2 \% ; \mathrm{P}<0,001)$, mas o sucesso angiográfico foi similar nos dois grupos (>99\%). No seguimento tardio, a incidência de óbito cardíaco $(6,4 \%$ vs. $2,7 \% ; \mathrm{P}<0,001)$ e de trombose de stent $(3,6 \%$ vs. $1,3 \% ; \mathrm{P}<0,001)$ foi significativamente maior no grupo IAM recente. O IAM recente permaneceu como preditor independente de trombose de stent na análise multivariada (RR 2,96, IC 95\% 1,62-5,41; $\mathrm{P}<0,001)$. Conclusões: Pacientes com IAM $<30$ dias tratados com SFs apresentaram pior prognóstico quando

\author{
ABSTRACT \\ Late Follow-up of Patients with Acute Myocardial \\ Infarction Treated with Drug-Eluting Stents \\ in the Daily Practice of the Real World - \\ A Sub-Analysis of the DESIRE Registry \\ (Drug-Eluting Stent In the REal World)
}

Background: Previous studies comparing drug-eluting stents (DES) and bare-metal stents suggest efficacy and safety of the DES in the treatment of patients with myocardial infarction (MI). However, the late evolution of patients with MI treated with DES in the daily practice remains unknown. Our goal was to investigate the late follow-up of patients with MI recently treated with DES in the real world. Methods: Between May 2002 and June 2009, 3,018 non-selected patients with indication for elective or urgent percutaneous coronary intervention have been consecutively treated with DES in a clinical institution. The 7-year follow-up was performed in $98 \%$ of these patients, with a median of 3.4 years. The patients were divided in two groups: patients with recent MI (< 30 days) and patients without recent MI. Results: Patients with recent $\mathrm{MI}$ had less co-morbidities, but more multiarterial disease $(68.1 \%$ vs. $60.7 \% ; \mathrm{P}<0.001)$, lesions with thrombus $(13.6 \%$ vs. $1.3 \% ; \mathrm{P}<0.001)$, TIMI flow $0 / 1$ $(8.3 \%$ vs. $1.1 \% ; \mathrm{P}<0.001)$, and moderate/severe LV dysfunction $(23.2 \%$ vs. $10.9 \%$; $\mathrm{P}<0.001)$ when compared with patients without $\mathrm{MI}$. The recent $\mathrm{MI}$ group received $1.6+0.8$ stents/patient and more glycoprotein Ilb/IIla inhibitors $(19.6 \%$ vs. $2 \% ; \mathrm{P}<0.001)$, but the angiographic success (> 99\%) was similar between groups. In the late follow-up, the incidence of cardiac death $(6.4 \%$ vs. $2.7 \% ; \mathrm{P}<0.001)$ and stent thrombosis $(3.6 \%$ vs. $1.3 \% ; \mathrm{P}<0.001)$ was significantly greater in the recent $\mathrm{MI}$ group. Recent $\mathrm{MI}$ remained an

\footnotetext{
1 Instituto de Ensino e Pesquisa - Hospital do Coração - Associação do Sanatório Sírio - São Paulo, SP, Brasil.

Correspondência: Ricardo A. Costa. Rua Abílio Soares, $250-12^{\circ}$ o andar - Paraíso - São Paulo, SP, Brasil - CEP 04005-000 E-mail: ricardoacosta@cardiol.br

Recebido em: 27/6/2011 • Aceito em: 23/8/2011
} 
comparados aos pacientes sem IAM recente, incluindo aumento significativo do óbito cardíaco até sete anos de acompanhamento, e ocorrência 2,5 vezes maior das taxas de trombose de stent.

DESCRITORES: Stents. Stents farmacológicos. Infarto do miocárdio. Angioplastia.
A introdução de stents farmacológicos (SFs) na prática clínica revolucionou o tratamento percutâneo da doença coronária. Vários estudos randomizados prévios comparando SFs e stents não-farmacológicos (SNFs) em lesões não-complexas demonstraram benefício significativo a favor dos SFs, principalmente pela marcante redução da formação de hiperplasia neointimal e de reestenose, e, consequentemente, da necessidade de revascularização da lesão-alvo (RLA). ${ }^{1,2}$ É importante salientar que não se evidenciou diferença significativa entre SFs e SNFs em relação ao perfil de segurança no acompanhamento clínico tardio. ${ }^{3}$ Logo, os SFs foram rapidamente incorporados à rotina da intervenção coronária percutânea (ICP) desde o início de sua comercialização, e sua utilização foi também disseminada em vários subgrupos de alto risco. ${ }^{4-9}$ Notavelmente, a eficácia e a segurança dos SFs têm sido confirmadas em cenários desafiadores ao intervencionista, incluindo lesões de morfologia complexa (bifurcações, tronco da coronária esquerda, trombo, oclusão, etc.) e quadros clínicos agudos. ${ }^{10,11}$ Especificamente, o impacto dos SFs no infarto agudo do miocárdio (IAM) foi avaliado em uma série de ensaios clínicos randomizados, em que se observou a superioridade desses dispositivos em relação aos SNFs (diminuição de $64 \%$ das taxas de revascularização do vaso-alvo) sem comprometimento da segurança tardia. ${ }^{12,13}$

Mesmo assim, estudos recentes de mundo real com SFs sugerem que a apresentação clínica de IAM recente pode estar associada a pior prognóstico, incluindo aumento das taxas de mortalidade, IAM e trombose de stent. ${ }^{14-16}$ Digno de nota é o fato de que a evolução tardia de pacientes com IAM tratados com SFs na prática diária permanece desconhecida. Portanto, este estudo teve como objetivo investigar o seguimento clínico muito tardio de pacientes com IAM recente $(<30$ dias $)$ tratados com SFs na prática do mundo real.

\section{MÉTODOS}

\section{Protocolo e população do estudo}

Os detalhes do protocolo e os resultados clínicos iniciais do registro Drug-Eluting Stent In the REal World (Registro DESIRE) já foram reportados previamente. ${ }^{17}$ Em resumo, o Registro DESIRE é um estudo clínico pros- independent predictor of stent thrombosis in multivariate analysis (HR 2.96, 95\% Cl 1.62-5.41; $\mathrm{P}<0,001)$. Conclusions: Patients with $\mathrm{MI}<30$ days treated with DES had a worse prognosis when compared with patients without recent MI, including a higher incidence of cardiac death and a 2.5 times greater occurrence of stent thrombosis rate up to 7 -year follow-up.

KEY-WORDS: Stents. Drug-eluting stents. Myocardial infarction. Angioplasty.

pectivo, não-randomizado, de braço único, com inclusão consecutiva de pacientes (inclusão em andamento), realizado em uma única instituição (Hospital do Coração/Associação do Sanatório Sírio - São Paulo, SP, Brasil), com o objetivo de investigar a evolução clínica tardia de pacientes tratados com SFs. Desde maio de 2002, a ICP com SFs tem sido utilizada como estratégia preferencial nos pacientes encaminhados para tratamento percutâneo de rotina ou emergência na referida instituição. São incluídos pacientes com pelo menos uma lesão com estenose $\geq 50 \%$ e anatomia favorável para ICP com implante de stent. Pelo protocolo, não existem limitações quanto ao número de lesões e/ ou vasos que podem ser tratados e quanto ao número de SFs implantados. O estudo está em adequação com a Declaração de Helsinque relacionada à investigação em humanos e foi aprovado pelo Comitê de Ética da instituição.

\section{Procedimento}

As ICPs foram realizadas de acordo com as diretrizes atuais ${ }^{18}$ e a estratégia final do procedimento foi deixada a critério do operador. Foram implantados SFs de primeira e segunda gerações: Cypher ${ }^{\mathrm{TM}}$ (Cordis, Miami, Estados Unidos), Taxus ${ }^{\mathrm{TM}}$ (Boston Scientific, Natick, Estados Unidos), Endeavor ${ }^{\mathrm{TM}}$ (Medtronic, Minneapolis, Estados Unidos), e Xience $V^{\mathrm{TM}} /$ Promus $^{\mathrm{TM}}$ (Abbott Laboratories, Abbot Park, Estados Unidos). Quando o resultado angiográfico imediato após implante do stent demonstrava subexpansão, uma pós-dilatação era realizada com cateter-balão de extensão mais curta que o stent, o qual era posicionado dentro das bordas do stent e insuflado a altas pressões (> $14 \mathrm{~atm}$ ). Implante de múltiplos stents e/ou tratamento de múltiplas lesões (por procedimento estagiado ou não) também eram permitidos. De maneira geral, em pacientes com síndrome coronária aguda (SCA), estudo angiográfico e ICP (quando indicada) eram realizados em até 48 horas do início da hospitalização.

O protocolo antitrombótico consistia na administração de dois agentes antiplaquetários: ácido acetilsalicílico (aspirina) e clopidogrel. Pré-tratamento com aspirina $500 \mathrm{mg}$ e clopidogrel $300 \mathrm{mg}$ foi realizado > 24 horas antes da intervenção para casos eletivos ou 600 mg de clopidogrel $>2$ horas antes do procedimento para 
SCA sem supradesnivelamento do segmento ST (SCASSST). No caso de ICP primária, a dose de ataque desses medicamentos era administrada no momento do procedimento. Após a intervenção, a terapia com aspirina era mantida indefinidamente, na dose de 100200 mg/dia, e o clopidogrel, na dose de 75 mg diários, era mantido, inicialmente, por 3-6 meses. A partir do final de 2006, seu uso foi estendido para 12 meses, de acordo com as recomendações do Food and Drug Administration (FDA), dos Estados Unidos. ${ }^{19}$ Durante o procedimento, heparina endovenosa era administrada (70-100 U/kg) para manter tempo de coagulação ativada > 250 segundos (ou > 200 segundos no caso de administração de inibidor da glicoproteína IIb/IIla). Eletrocardiograma (ECG) com 12 derivações era obtido antes, imediatamente após e 24 horas depois do procedimento. Exames laboratoriais incluíam enzimas cardíacas (creatina fosfoquinase - CPK e creatina fosfoquinase fração $\mathrm{MB}-\mathrm{CK}-\mathrm{MB}$ massa) pré-procedimento ( $<24$ horas), 18-24 horas pós-procedimento, e diariamente, em caso de alteração, até a alta hospitalar.

\section{Análise angiográfica}

A análise angiográfica qualitativa incluiu a avaliação do território miocárdico acometido, definido como a porção do músculo cardíaco irrigada por uma artéria epicárdica maior e seus respectivos ramos. Em relação à análise quantitativa, após a administração intracoronária de nitrato $(50-200 \mu \mathrm{g})$, estudos seriados foram obtidos incluindo angiografia pré e pós-procedimento. As análises de angiografia coronária quantitativa (ACQ) foram realizadas após o procedimento (off-line) por operadores experientes, utilizando um sistema de análise quantitativa com detecção semiautomática das bordas luminais - QuantcorQCA-ACOM.PC versão 4.0 (Siemens, Munique, Alemanha). O diâmetro luminal mínimo (DLM) e o diâmetro de referência do vaso (DRV), obtido pela média dos segmentos 5-10 $\mathrm{mm}$ proximal e distal da lesão-alvo, foram utilizados para calcular o diâmetro de estenose [DE $=(1-\mathrm{DLM} / \mathrm{DRV}) \times 100]$. Ganho imediato foi definido como a diferença do DLM pré e pós-procedimento (DLM pós-procedimento - DLM pré-procedimento).

\section{Objetivos, definições e seguimento clínico}

O objetivo primário do estudo foi a ocorrência de eventos cardíacos adversos maiores (ECAM) no seguimento clínico tardio ( $>6$ meses). O desfecho composto de ECAM incluía: morte cardíaca, IAM e RLA. Como regra, todas as mortes foram consideradas cardíacas, a não ser que uma causa não-cardíaca pudesse ser claramente estabelecida por estudo clínico e/ou patológico. O diagnóstico de IAM foi baseado no aparecimento de nova onda $\mathrm{Q}$ patológica em $\geq 2$ derivações contíguas no ECG e/ou elevação da CK-MB massa > 3 vezes o limite superior da normalidade. As tromboses de stent foram definidas de acordo com o Academic
Research Consortium (ARC) ${ }^{20}$ em: definitiva (presença de SCA com confirmação angiográfica ou anatomopatológica da oclusão do stent); provável (ocorrência de morte súbita $<30$ dias pós-procedimento índice ou IAM no território miocárdico tratado, sem confirmação angiográfica da oclusão do stent); e possível (ocorrência de morte súbita > 30 dias após o procedimento índice). As tromboses de stent ainda foram classificadas de acordo com a ocorrência temporal, incluindo: fase aguda ( $\leq 24$ horas do procedimento), fase subaguda (entre 24 horas e 30 dias), fase tardia (entre 1 mês e 12 meses), e fase muito tardia (> 12 meses). O sucesso angiográfico foi definido como: fluxo final TIMI 3, ausência de dissecções e estenose residual $<20 \%$ pela ACQ. O seguimento clínico foi realizado 1 mês, 6 meses e 12 meses após o procedimento, e, a seguir, anualmente, consistindo de visita médica agendada ou contato telefônico, realizados segundo protocolo pré-definido. Todos os eventos adversos reportados, incluindo trombose de stent, foram independentemente adjudicados por um Comitê de Eventos Clínicos, incluindo três experientes profissionais das áreas de cardiologia clínica e invasiva.

O fluxograma de inclusão de pacientes no Registro DESIRE é mostrado na Figura 1. No total 3.222 pacientes foram incluídos entre maio de 2002 e novembro de 2009. Para o objetivo da análise atual, os pacientes com no mínimo 6 meses de seguimento $(\mathrm{n}=3.018$, inclusão entre maio de 2002 e junho de 2009) foram divididos em dois grupos, de acordo com a apresentação clínica de IAM recente (definido como IAM ocorrido < 30 dias do procedimento índice): grupo 1 , IAM recente $(\mathrm{n}=476)$; e grupo 2, sem IAM recente $(\mathrm{n}=2.542)$. Os dois grupos tiveram os resultados comparados.

\section{Análise estatística}

As variáveis são apresentadas como frequências (\% do total) ou médias \pm desvios padrão. As variáveis categóricas foram comparadas pelo teste de qui-quadrado, e, quando indicado, pelo teste exato de Fisher. As variáveis quantitativas foram comparadas pelo teste $t$ de Student. A ocorrência de eventos adversos em função do tempo foi descrita por meio de curvas de Kaplan-Meier, comparadas pelo teste de log-rank. Para identificar fatores associados ao desenvolvimento de trombose de stent foi utilizado o modelo de riscos proporcionais de Cox. Inicialmente foram avaliadas todas as variáveis em análises bivariadas para depois serem analisadas de forma múltipla, considerando nível de significância de $10 \%$. A permanência das variáveis no modelo múltiplo foi baseada no teste de razão de verossimilhanças e relevância clínica. Os resultados do modelo de Cox foram apresentados em razões de risco (RR) e respectivos intervalos de confiança (IC) de 95\%. Valor de $\mathrm{P}<0,05$ foi considerado significativo. As análises foram realizadas com os programas estatísticos SPSS versão 16.0 (SPSS Inc., Chicago, Estados Unidos) e STATA versão 10 (StataCorp LP, College Station, Estados Unidos). 


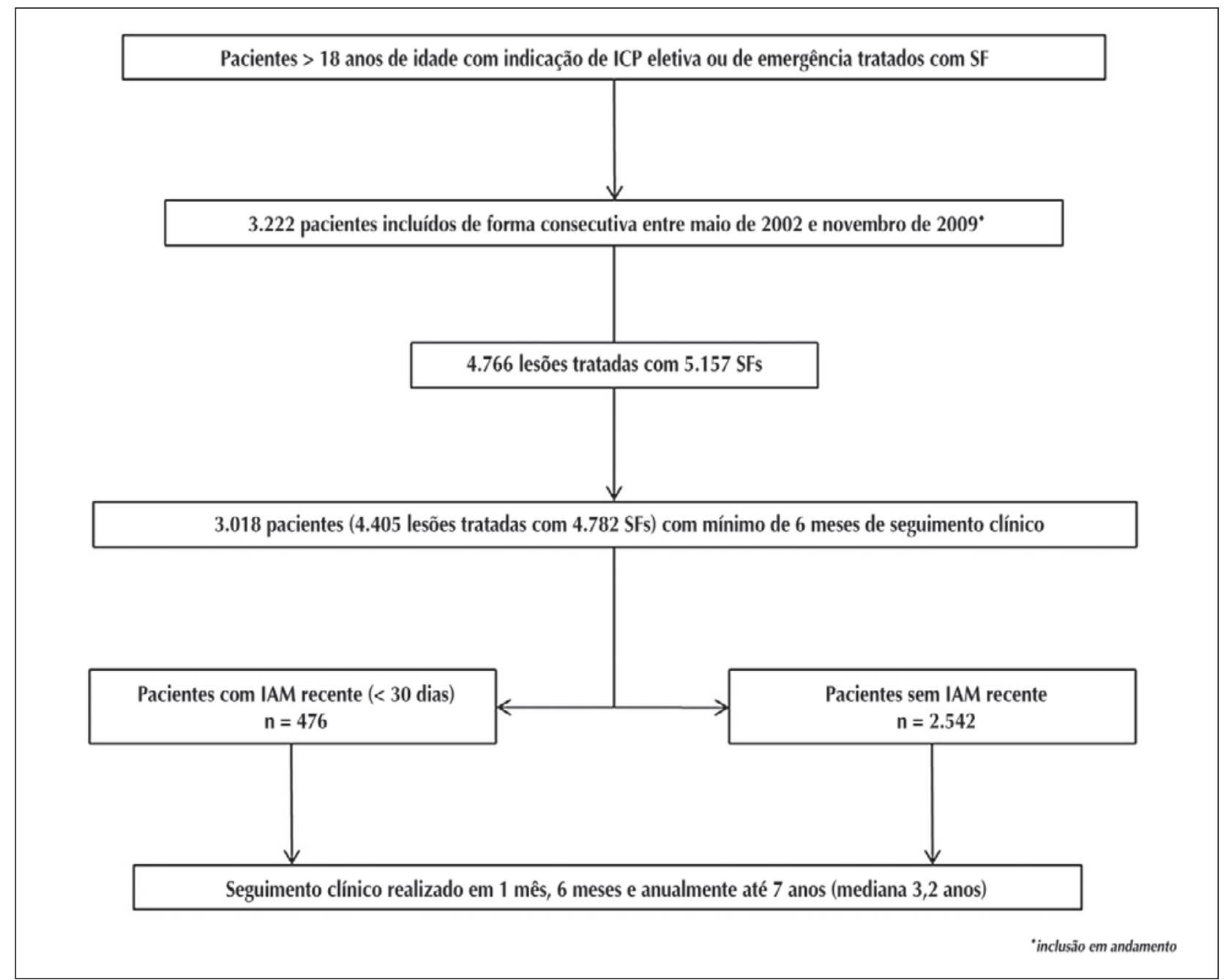

Figura 1 - Fluxograma de inclusão de pacientes do Registro DESIRE. IAM = infarto agudo do miocárdio; ICP = intervenção coronária percutânea; $\mathrm{n}=$ número de pacientes; $\mathrm{SF}=$ stent farmacológico.

\section{RESULTADOS}

\section{Características basais}

Comparativamente ao grupo 2, o grupo 1 apresentava menor prevalência de diabetes melito, hipertensão arterial sistêmica, dislipidemia, história familiar de doença arterial coronária e revascularização prévia; no entanto, o grupo 1 tinha significativamente mais tabagistas, IAM prévio, e insuficiência renal (Tabela 1). Os dados angiográficos estão apresentados na Tabela 2. A artéria descendente anterior foi o vaso-alvo mais tratado em ambos os grupos, incluindo seu segmento proximal em $21,2 \%$ no grupo 1 vs. $19,9 \%$ no grupo $2(\mathrm{P}=0,44)$. O grupo 1 também tinha mais doença multiarterial, lesões complexas (incluindo lesões com trombo) e fluxo coronário comprometido. Adicionalmente, 23,2\% dos pacientes no grupo 1 apresentavam disfunção moderada/grave do ventrículo esquerdo vs. $10,9 \%$ no grupo $2(\mathrm{P}<0,001)$.

\section{Procedimento e angiografia quantitativa}

Foi tratada 1,4 $\pm 0,7$ lesão por paciente no grupo 1 vs. $1,5 \pm 0,7$ no grupo $2(P=0,13)$. Em relação aos medicamentos em uso na admissão, o grupo 1 recebia mais betabloqueadores (55,7\% vs. 41,9\%; P < 0,001), antianginosos (71,6\% vs. 55,3\%; P < 0,001) e estatinas (71,4\% vs. 59,8\%; P < 0,001), comparativamente ao grupo 2; no entanto, a maioria dos pacientes em ambos os grupos recebia terapêutica antiplaquetária dupla, incluindo aspirina $(98,3 \%$ vs. 98,5\%; $\mathrm{P}=0,61)$ e tienopiridínico $(99,4 \%$ vs. 99,8\%; $P=0,09)$. Já os agentes inibidores da glicoproteína IIb/IIla foram administrados em $19,6 \%$ no grupo 1 vs. $2 \%$ no grupo $2(P<0,001)$.

As lesões do grupo 1 receberam significativamente mais pré e pós-dilatação (Tabela 3). Pela ACQ, as lesões do grupo 1 eram mais extensas e com estenoses mais significativas, quando comparadas às do grupo 2 (Tabela 4). Consequentemente, o grupo 1 recebeu dispo- 
TABELA 1

Características clínicas basais

\begin{tabular}{|c|c|c|c|}
\hline Variável & $\begin{array}{c}\text { Grupo } 1 \\
(n=476)\end{array}$ & $\begin{array}{c}\text { Grupo 2 } \\
(\mathrm{n}=2.542)\end{array}$ & Valor de $\mathbf{P}$ \\
\hline Média de idade, anos & $63 \pm 12,8$ & $64,2 \pm 11,1$ & 0,06 \\
\hline Sexo feminino, $\%$ & 21,2 & 23,9 & 0,3 \\
\hline Diabetes melito, \% & 25 & 29,7 & 0,04 \\
\hline Dependente de insulina & 5 & 5,7 & 0,55 \\
\hline Hipertensão arterial sistêmica, \% & 68,1 & 77,5 & $<0,001$ \\
\hline Dislipidemia, \% & 53,6 & 63 & $<0,001$ \\
\hline Tabagismo atual, \% & 36,3 & 30,3 & 0,009 \\
\hline História familiar de DAC, \% & 34,5 & 40,3 & 0,02 \\
\hline IAM prévio*, \% & 30,9 & 20,6 & $<0,001$ \\
\hline ICP prévia, \% & 20 & 26 & 0,005 \\
\hline CRM prévia, \% & 14,9 & 27,7 & $<0,001$ \\
\hline AVE prévio, \% & 3,6 & 2,1 & 0,06 \\
\hline Insuficiência renal ${ }^{+}, \%$ & 15,5 & 10,3 & 0,003 \\
\hline Elevação do segmento ST, \% & 21,2 & - & - \\
\hline Angioplastia primária, \% & 14,5 & - & - \\
\hline Terapia com trombolítico ${ }^{\ddagger}, \%$ & 12 & - & - \\
\hline
\end{tabular}

${ }^{*}>30$ dias. ${ }^{+}$Creatinina sérica basal $>1,5 \mathrm{mg} / \mathrm{dl} .{ }^{*}$ Inclui angioplastia de resgate.

$\mathrm{AVE}=$ acidente vascular encefálico; $\mathrm{CRM}=$ cirurgia de revascularização do miocárdio; $\mathrm{DAC}=$ doença arterial coronária; IAM = infarto agudo do miocárdio; ICP = intervenção coronária percutânea; $\mathrm{n}$ = número de pacientes.

TABELA 2

Dados angiográficos

\begin{tabular}{|c|c|c|c|}
\hline Variável & $\begin{array}{c}\text { Grupo } 1 \\
\text { (476 pacientes/ } \\
678 \text { lesões) }\end{array}$ & $\begin{array}{c}\text { Grupo } 2 \\
\text { (2.542 pacientes/ } \\
3.727 \text { lesões) }\end{array}$ & Valor de $\mathbf{P}$ \\
\hline \multicolumn{4}{|l|}{ Território miocárdico acometido, \% } \\
\hline Artéria coronária descendente anterior & 45,7 & 42,2 & 0,11 \\
\hline Artéria coronária circunflexa & 25,6 & 29,3 & 0,06 \\
\hline Coronária direita & 27,3 & 27,2 & 0,94 \\
\hline Tronco de coronária esquerda & 1,4 & 1,3 & 0,77 \\
\hline Doença multiarterial*, \% & 68,1 & 60,7 & $<0,001$ \\
\hline Excentricidade, \% & 28,8 & 25,6 & 0,08 \\
\hline Calcificação moderada/grave, \% & 26,8 & 26,3 & 0,76 \\
\hline Trombo, \% & 13,6 & 1,3 & $<0,001$ \\
\hline Ponte de safena, \% & 4,1 & 5,8 & 0,08 \\
\hline Localização ostial, \% & 4 & 5 & 0,27 \\
\hline Reestenose intrastent, \% & 3,1 & 4,6 & 0,09 \\
\hline Lesão tipo B2/C $\mathrm{C}^{+}, \%$ & 70,3 & 66,3 & 0,06 \\
\hline \multicolumn{4}{|l|}{ Fluxo TIMI, \% } \\
\hline Grau 0 & 6,8 & 0,6 & $<0,001$ \\
\hline Grau 1 & 1,5 & 0,5 & 0,004 \\
\hline Grau 2 & 4,1 & 1,3 & $<0,001$ \\
\hline Grau 3 & 87,6 & 97,7 & $<0,001$ \\
\hline Fração de ejeção do VE < 40\%, \% & 23,2 & 10,9 & $<0,001$ \\
\hline
\end{tabular}

* Acometimento de 2 ou 3 vasos. ${ }^{+}$De acordo com a classificação do American College of Cardiology/American Heart Association. $\mathrm{VE}=$ ventrículo esquerdo. 
TABELA 3

Características do procedimento

\begin{tabular}{|c|c|c|c|}
\hline Variável & $\begin{array}{c}\text { Grupo } 1 \\
\text { (476 pacientes/ } \\
678 \text { lesões) }\end{array}$ & $\begin{array}{c}\text { Grupo } 2 \\
\text { (2.542 pacientes/ } \\
3.727 \text { lesões) }\end{array}$ & Valor de $\mathbf{P}$ \\
\hline Pré-dilatação, \% & 57,2 & 49,9 & $<0,001$ \\
\hline > 1 stent implantado, \% & 40,8 & 43,5 & 0,28 \\
\hline Número de stents implantados por paciente, \% & $1,6 \pm 0,8$ & $1,6 \pm 0,8$ & $>0,99$ \\
\hline Número de stents implantados por lesão, \% & $1,1 \pm 0,4$ & $1,1 \pm 0,3$ & $>0,99$ \\
\hline \multicolumn{4}{|l|}{ Tipo de SF implantado, \% } \\
\hline Cypher ${ }^{\mathrm{TM}}$ & 81,6 & 84,6 & 0,05 \\
\hline Endeavor $^{\mathrm{TM}}$ & 4,2 & 2,9 & 0,06 \\
\hline Taxus $^{\mathrm{TM}}$ & 9,7 & 9,9 & 0,92 \\
\hline Xience $\mathrm{V}^{\mathrm{TM}} /$ Promus $^{\mathrm{TM}}$ & 4,1 & 2,2 & 0,003 \\
\hline Outros & 0,4 & 0,4 & 0,88 \\
\hline Extensão nominal do stent, mm & $20,1 \pm 6,2$ & $19,4 \pm 5,9$ & 0,005 \\
\hline Diâmetro nominal do stent, $\mathrm{mm}$ & $2,9 \pm 0,4$ & $2,9 \pm 0,4$ & $>0,99$ \\
\hline Pressão de implante do stent, atm & $14,3 \pm 2,7$ & $14,4 \pm 3$ & 0,39 \\
\hline Pós-dilatação, \% & 56,2 & $48, \overline{8}$ & $<0,001$ \\
\hline Pressão máxima durante pós-dilatação, atm & $15,2 \pm 2$ & $15,5 \pm 3,7$ & 0,04 \\
\hline Fluxo TIMI final grau 3, \% & $99, \overline{6}$ & 99,8 & 0,12 \\
\hline Sucesso angiográfico, \% & 99,6 & 99,3 & 0,41 \\
\hline
\end{tabular}

TABELA 4

Angiografia coronária quantitativa pré e pós-procedimento

\begin{tabular}{lccc}
\hline Variável & $\begin{array}{c}\text { Grupo } \mathbf{1} \\
\text { (678 lesões) }\end{array}$ & $\begin{array}{c}\text { Grupo } \mathbf{2} \\
(\mathbf{3 . 7 2 7} \text { lesões })\end{array}$ & Valor de $\mathbf{P}$ \\
\hline Pré-procedimento & & & \\
$\quad$ Extensão da lesão, mm & $17,8 \pm 8,9$ & $16,7 \pm 8,4$ & 0,003 \\
DRV, mm & $2,78 \pm 0,45$ & $2,77 \pm 0,48$ & 0,6 \\
DLM, mm & $0,8 \pm 0,54$ & $0,96 \pm 1,19$ & $<0,001$ \\
DE, \% & $71,1 \pm 13,1$ & $66,7 \pm 10,9$ & $<0,001$ \\
Pós-procedimento & $2,79 \pm 0,65$ & $2,8 \pm 0,76$ & 0,72 \\
DLM, mm & $4,4 \pm 3,5$ & $4,5 \pm 4$ & 0,5 \\
DE, \% & $1,99 \pm 0,56$ & $1,86 \pm 0,48$ & $<0,001$ \\
Ganho imediato, mm & & \\
\hline DE $=$ diâmetro de estenose; DLM = diâmetro luminal mínimo; DRV = diâmetro de referência do vaso. \\
\hline
\end{tabular}

sitivos mais longos $(P=0,005)$; mesmo assim, o sucesso angiográfico foi similar nos dois grupos (> 99\%). Durante a fase hospitalar, todos os pacientes foram mantidos com terapia antiplaquetária dupla e a prescrição foi mantida na alta hospitalar, de acordo com o protocolo.

\section{Eventos clínicos}

$\mathrm{Na}$ fase intra-hospitalar, a mortalidade cardíaca foi significativamente aumentada no grupo 1 quando comparada à do grupo $2(1,3 \%$ vs. $0,1 \%$, respectivamente;
$P<0,001$ ), mas as taxas de IAM (reinfarto no grupo 1) foram semelhantes em ambos os grupos (Tabela 5). Similarmente, no seguimento clínico tardio até 7 anos (realizado em 98\%; mediana: 3,2 anos), as taxas cumulativas de morte cardíaca foram maiores no grupo $1(6,4 \%$ vs. $2,7 \%$; $P<0,001)$; entretanto, não se observou diferença significativa nas taxas cumulativas de IAM, RLA, e ECAM. As curvas de Kaplan-Meier de sobrevida livre de óbito cardíaco, IAM e ECAM até 7 anos de seguimento são mostradas na Figura $2 \mathrm{~A}, \mathrm{~B}$ e C. 
TABELA 5

Eventos clínicos adversos

\begin{tabular}{|c|c|c|c|c|c|c|}
\hline \multirow[b]{2}{*}{ Evento } & \multicolumn{3}{|c|}{ Fase intra-hospitalar } & \multicolumn{3}{|c|}{ Seguimento tardio $^{\dagger}$} \\
\hline & Grupo 1 & Grupo 2 & Valor de $\mathbf{P}$ & Grupo 1 & Grupo 2 & Valor de $P$ \\
\hline Morte cardíaca, \% & 1,3 & 0,1 & $<0,001$ & 6,4 & 2,7 & $<0,001$ \\
\hline IAM, \% & $3,4^{*}$ & 4 & 0,56 & $6,4^{*}$ & 6,1 & 0,79 \\
\hline Com onda Q & $0,4^{*}$ & 0,1 & & $1,7^{*}$ & 1,1 & \\
\hline Sem onda Q & $2,9^{*}$ & 3,9 & & $4,7^{*}$ & 5 & \\
\hline RLA, \% & 0 & 0 & NA & 3,2 & 4,7 & 0,16 \\
\hline ECAM, \% & 4,6 & 4,1 & 0,49 & 16 & 13,5 & 0,16 \\
\hline TS, \% & 0 & 0 & $\mathrm{NA}$ & 3,6 & 1,3 & $<0,001$ \\
\hline
\end{tabular}

* Taxas de reinfarto. ${ }^{+}$Incidência cumulativa de eventos.

ECAM = eventos cardíacos adversos maiores; IAM = infarto agudo do miocárdio; NA = não aplicável; RLA = revascularização da lesão-alvo; TS = trombose de stent.

No total, ocorreram 48 tromboses de stent durante o período de acompanhamento, e a taxa de trombose de stent foi significativamente maior no grupo 1 $(3,6 \%$ vs. $1,3 \% ; P<0,001)$. As tromboses, em sua maioria, foram definitivas (32/48), e corresponderam a $60 \%$ e $70 \%$ do total de tromboses de stent nos grupos 1 e 2, respectivamente. Em relação à classificação temporal da trombose de stent, não foram observados eventos na fase aguda; já nas fases subaguda, tardia e muito tardia, as taxas foram de $1,1 \%$ vs. $0,2 \%(P=0,007)$, $1,5 \%$ vs. $0,5 \%(P=0,01)$, e $1,1 \%$ vs. $0,6 \%(P=0,16)$ para os grupos 1 e 2 , respectivamente. A curva de incidência cumulativa de trombose de stent até 7 anos de seguimento é mostrada na Figura 2 D.

Os preditores independentes significativos de trombose de stent identificados no modelo multivariado foram: IAM recente, tabagismo atual, diabetes melito, excentricidade, calcificação moderada/grave, e estenose residual (Tabela 6). Finalmente, considerando-se apenas as tromboses de stent definitivas e prováveis (ARC), as taxas de trombose de stent permaneceram significativamente aumentadas no grupo $1(2,1 \%$ vs. $0,9 \% ; P=0,02)$.

\section{IAM com supradesnivelamento do segmento ST}

No grupo 1, 101 dos 476 pacientes (21,2\%) tinham IAM com elevação do segmento ST (IAMCSST). Destes, 55 se apresentaram $<24$ horas após o início dos sintomas e foram submetidos a ICP primária.

O óbito cardíaco na fase hospitalar dos pacientes com IAMCSST foi de 3\% vs. 0,8\% nos pacientes com IAM sem supradesnivelamento do segmento ST (IAMSSST) $(\mathrm{P}=0,07)$. No acompanhamento tardio, as taxas cumulativas de morte cardíaca ( $5 \%$ vs. $6,8 \% ; P=0,57)$ e reinfarto $(5,9 \%$ vs. $6,5 \% ; P=0,91)$ foram similares, comparando IAMCSST e IAMSSST, respectivamente. Com relação à incidência de trombose de stent, os pacientes com IAMCSST tiveram 3\% de eventos $(n=3)$, incluindo 3 tromboses definitivas, que ocorreram na fase subaguda $(\mathrm{n}=2)$ e na fase tardia $(\mathrm{n}=1)$.

\section{DISCUSSÃo}

Na análise atual, o IAM recente esteve significativamente associado a maior mortalidade cardíaca tanto precoce como tardia e a aumento das taxas de trombose de stent, comparativamente à população sem IAM. Tal achado impactou de forma significativa a ocorrência de ECAM no seguimento até 7 anos.

De maneira geral, a apresentação clínica de IAM como indicador de ICP com SF tem sido relacionada a pior prognóstico no mundo real. Montalescot et al. ${ }^{14}$ reportaram os resultados iniciais de 7.962 pacientes do mundo real submetidos a ICP com SF em 144 centros em 23 países no estudo Registry on IntraVenous anticoagulation In the Elective and primary Real world of Angioplasty (RIVIERA). Nesse estudo, a clínica de IAM (IAMCSST e IAMSSST) foi preditora de morte e IAM. Com relação à trombose de stent, vários estudos recentes com SFs têm demonstrado associação significativa entre a ICP no IAM e a ocorrência de trombose de stent. ${ }^{16,21}$ De fato, o IAM recente foi identificado como um dos mais poderosos preditores de trombose de stent no Registro DESIRE (RR 2,96). Embora os motivos de tal associação ainda não estejam completamente elucidados, alguns mecanismos têm sido propostos, incluindo:

- desenvolvimento de aposição incompleta das hastes $(\mathrm{AIH})$ do stent decorrente de lise posterior do trombo aderido à parede 22 (nos pacientes com IAM, a presença, visível ou não, de trombo na lesão culpada é marcante, e a $\mathrm{AlH}$ parece ser mais pronunciada nos casos de grande acúmulo de trombo; ${ }^{22}$ estudos de relatos de caso, análise histopatológica e séries clínicas têm evidenciado a AIH adquirida tardiamente como preditor de trombose muito tardia de SF); ${ }^{23-25}$ 


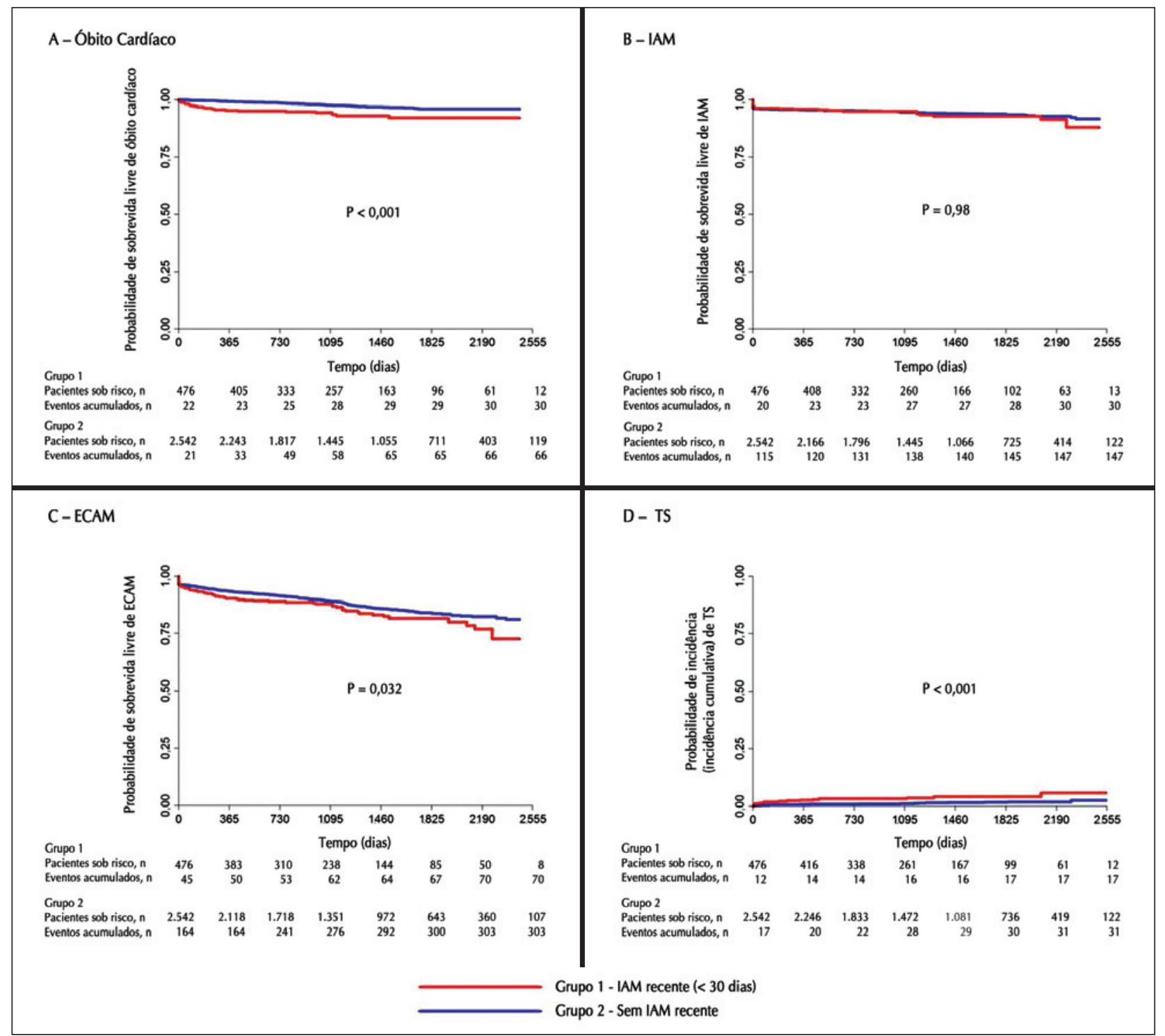

Figura 2 - Estimativas de sobrevida livre de eventos no seguimento tardio (até 7 anos) pelo método de Kaplan-Meier. Em A, curvas de sobrevida livre de óbito cardíaco. Em B, curvas de sobrevida livre de infarto agudo do miocárdio. Em C, curvas de sobrevida livre de eventos cardíacos adversos maiores. Em D, incidência cumulativa de trombose de stent. ECAM = eventos cardíacos adversos maiores; IAM = infarto agudo do miocárdio; TS = trombose de stent.

- reendotelização tardia do SF;26 e

- lesão com atividade inflamatória contínua (não cessa com implante de SF), a qual pode apresentar maior potencial trombogênico.

Nesta análise, os pacientes do grupo 1 apresentaram maior complexidade das lesões coronárias, incluindo presença de trombo 10 vezes maior que o grupo 2 $(P<0,001$ ) e oclusão do vaso (fluxo TIMI 0/1) em 8,3\% $(P<0,001)$. Curiosamente, tais achados não impactaram os resultados angiográficos. Tanto o trombo como a interrupção do fluxo coronário são clássicos preditores angiográficos de complicações intraprocedimento e pior prognóstico clínico. ${ }^{22,27-29}$ Logo, o fato de um quinto dos pacientes do grupo 1 terem recebido inibidores da glicoproteína Ilb/Illa poderia $(\mathrm{P}<0,001)$, pelos menos em parte, explicar esses resultados. De maneira geral, esses fármacos atuam na via final do processo de agregação plaquetária por meio do bloqueio seletivo dos receptores de superfície das plaquetas, impedindo dessa forma a formação do trombo. ${ }^{30}$ Notavelmente, a restauração de fluxo TIMI 3 na artéria culpada no IAM pode não refletir perfusão miocárdica adequada. Em uma subanálise do estudo Controlled Abciximab and Device Investigation to Lower Late Angioplasty Complications (CADILLAC), Costantini et al. ${ }^{31}$ demonstraram que a perfusão miocárdica (blush) normal pós-ICP primária só estava presente em $17,4 \%$ dos pacientes com fluxo 
TABELA 6

Modelo multivariado identificando preditores independentes de trombose de stent

\begin{tabular}{lccc}
\hline Variável & RR & IC 95\% & Valor de P \\
\hline IAM recente $(<30$ dias) & 2,96 & $1,62-5,41$ & $<0,001$ \\
Tabagismo atual & 2,52 & $1,13-4,48$ & 0,021 \\
Diabetes melito & 1,84 & $1,01-3,34$ & 0,046 \\
Excentricidade da lesão & 2,78 & $1,38-5,62$ & 0,004 \\
Calcificação significativa & 2,71 & $1,33-5,54$ & 0,006 \\
Estenose residual & $1,06^{*}$ & $1,03-1,09$ & $<0,001$ \\
\hline
\end{tabular}

* RR estimado para cada unidade porcentual da referida variável.

IAM = infarto agudo do miocárdio; IC 95\% = intervalo de confiança de 95\%; RR = razão de risco.

final TIMI 3. Notavelmente, esse biomarcador foi um preditor de sobrevida nesse estudo, sendo a mortalidade em um ano de 1,4\% com blush normal vs. 4,1\% com blush reduzido vs. $6,2 \%$ com blush ausente $(P=0,01)$. Em duas análises reportadas por de Lemos et al. ${ }^{32}$ (TIMI 14) e Petronio et al. $^{33}$, a administração de inibidores da glicoproteína IIb/IIla durante a terapia de reperfusão no IAM otimizou o fluxo epicárdico e a perfusão miocárdica. Por último, em subanálise do estudo Enhanced Myocardial Efficacy and Removal by Aspiration of Liberated Debris (EMERALD), Lansky et al. ${ }^{34}$ demonstraram correlação significativa entre grau de perfusão miocárdica, resolução do segmento ST e disfunção ventricular em pacientes submetidos a ICP primária ou de resgate. Neste estudo, a perfusão miocárdica não foi avaliada de maneira sistemática, mas a análise de função ventricular esquerda demonstrou disfunção moderada/grave em quase um quarto dos pacientes do grupo $1(\mathrm{P}<0,001)$

Estudos prévios randomizados envolvendo pacientes com IAM sugerem benefício dos SFs vs. SNFs semelhante ao observado em cenários menos complexos. ${ }^{12,35,36}$ Tais achados incluem diminuições significativas das taxas de RLA, sem comprometimento da segurança (taxas semelhantes de óbito/IAM). ${ }^{12}$ No entanto, estudo observacionais de mundo real comparando SFs vs. SNFs em pacientes com IAM têm demonstrado resultados conflitantes..$^{37,38}$ Dessa forma, Mauri et al. ${ }^{13}$ conduziram um estudo envolvendo grande coorte de pacientes não-selecionados com apresentação clínica de IAM (IAMCSST e IAMSSST) submetidos a ICP (SFs e SNFs) em 21 hospitais no Estado de Massachusetts, Estados Unidos, entre abril de 2001 e setembro de 2004. No geral, os pacientes tratados com SF receberam stent Cypher $^{\mathrm{TM}}$ em $71 \%$, Taxus ${ }^{\mathrm{TM}}$ em $27 \%$, e ambos em $2 \%$. Através de ajuste estatístico de escore de propensão para minimizar a ação de fatores confundidores e diferenças não-balanceadas entre os grupos (pacientes não eram randomizados), foram identificados 2.570 pares tratados com SFs vs. SNFs, e a mortalidade total (desfecho primário) ao final de dois anos foi de 10,7\% com SFs vs. $12,8 \%$ com SNFs $(P=0,02)$. Quando considerados apenas os pacientes com IAMCSST, a mortalidade total foi de $8,5 \%$ com SFs vs. 11,6\% com SNFs ( $P=0,008)$; em relação aos pacientes com IAMSSST, a mortalidade total foi de 12,8\% com SFs vs. 15,6\% com SNFs $(P=0,04)$. Com relação à ocorrência de reinfarto, as taxas foram de $9,3 \%$ vs. $10,8 \%(P=0,09)$ na população total, de $7,4 \%$ vs. $8,5 \%(P=0,34)$ no IAMCSST, e de $10,8 \%$ vs. $14,1 \%(P=0,02)$ no IAMSSST para SFs vs. SNFs, respectivamente.

Resultados semelhantes foram encontrados neste estudo, que incluiu pacientes consecutivamente tratados com SFs desde maio de 2002. No Registro DESIRE, a maioria dos pacientes recebeu o SF Cypher ${ }^{\mathrm{TM}}(83,1 \%)$, seguido de Taxus ${ }^{\mathrm{TM}}$ em 9,2\% e outro SF em 7,7\%. Nos pacientes do grupo 1 , a mortalidade total e o reinfarto foram de $10,7 \%$ e $6,4 \%$ no seguimento de 3,2 anos; adicionalmente, considerando-se os subgrupos de IAM, as taxas foram de $7,9 \%$ e $5,9 \%$ no IAMCSST e de $11,4 \%$ e $6,5 \%$ no IAMSSST, respectivamente. Diferentemente do estudo de Mauri et al. ${ }^{13}$, foram incluídos apenas pacientes tratados com SF no Registro DESIRE; ${ }^{17}$ mesmo assim, especulou-se que os achados do estudo de Massachusetts poderiam estar associados a redução de reestenose e, consequentemente, procedimentos adicionais com SF. ${ }^{39,40}$ De qualquer maneira, existem poucos dados na literatura referentes à eficácia e à segurança tardia dos SFs em pacientes com IAM tratados na prática diária; portanto, são necessários novos estudos comparativos com número adequado de pacientes e acompanhamento muito tardio para definir o impacto dos SFs nesse subgrupo complexo.

\section{CONCLUSÕES}

Pacientes do mundo real com apresentação clínica de IAM recente $(<30$ dias) submetidos a implante de SFs apresentaram taxas significativamente aumentadas de óbito cardíaco no seguimento tardio de até 7 anos, quando comparados aos pacientes sem IAM recente. Tal achado foi mais pronunciado no subgrupo de pacientes com IAMCSST, os quais apresentaram taxa de mortalidade cardíaca intra-hospitalar 70\% maior 
que aqueles com IAMSSST. Adicionalmente, a ocorrência de trombose de stent foi maior nos pacientes com IAM recente, em decorrência, principalmente, de elevações significativas das taxas de trombose de stent subaguda e tardia. Finalmente, considerando-se a população total do estudo, a apresentação clínica de IAM recente permaneceu como um dos mais poderosos preditores de trombose de stent.

\section{AGRADECIMENTOS}

Os autores agradecem a dedicação, a competência e a significativa colaboração dos seguintes profissionais envolvidos no Registro DESIRE: Cantídio Campos, Fábio Kawasetsu, Luciana Fernandes Alves, Mariana T. Carballo, Valéria Ranieri, e Wagner Pinto.

\section{CONFLITO DE INTERESSES}

Os autores declaram não haver conflito de interesses relacionado a este manuscrito.

\section{REFERÊNCIAS}

1. Moses JW, Leon MB, Popma JJ, Fitzgerald PJ, Holmes DR, $\mathrm{O}^{\prime}$ Shaughnessy $\mathrm{C}$, et al. Sirolimus-eluting stents versus standard stents in patients with stenosis in a native coronary artery. $\mathrm{N}$ Engl J Med. 2003;349(14):1315-23.

2. Stone GW, Ellis SG, Cox DA, Hermiller J, O'Shaughnessy C, Mann JT, et al. A polymer-based, paclitaxel-eluting stent in patients with coronary artery disease. N Engl J Med. 2004; 350(3):221-31.

3. Stone GW, Moses JW, Ellis SG, Schofer J, Dawkins KD, Morice MC, et al. Safety and efficacy of sirolimus- and paclitaxel-eluting coronary stents. N Engl J Med. 2007;356(10): 998-1008.

4. Sousa JE, Costa MA, Abizaid A, Sousa AG, Feres F, Mattos LA, et al. Sirolimus-eluting stent for the treatment of in-stent restenosis: a quantitative coronary angiography and threedimensional intravascular ultrasound study. Circulation. 2003; 107(1):24-7.

5. Lemos PA, Serruys PW, van Domburg RT, Saia F, Arampatzis CA, Hoye A, et al. Unrestricted utilization of sirolimuseluting stents compared with conventional bare stent implantation in the "real world": the Rapamycin-Eluting Stent Evaluated At Rotterdam Cardiology Hospital (RESEARCH) registry. Circulation. 2004;109(2):190-5.

6. Ong AT, Serruys PW, Aoki J, Hoye A, van Mieghem CA, Rodriguez-Granillo GA, et al. The unrestricted use of paclitaxelversus sirolimus-eluting stents for coronary artery disease in an unselected population: one-year results of the Taxus-Stent Evaluated at Rotterdam Cardiology Hospital (T-SEARCH) registry. J Am Coll Cardiol. 2005;45(7):1135-41.

7. Valgimigli $M$, van Mieghem CA, Ong AT, Aoki J, Granillo GA, McFadden EP, et al. Short- and long-term clinical outcome after drug-eluting stent implantation for the percutaneous treatment of left main coronary artery disease: insights from the Rapamycin-Eluting and Taxus Stent Evaluated At Rotterdam Cardiology Hospital registries (RESEARCH and T-SEARCH). Circulation. 2005;111(11):1383-9.

8. Lemos PA, Lee CH, Degertekin M, Saia F, Tanabe K, Arampatzis $\mathrm{CA}$, et al. Early outcome after sirolimus-eluting stent implantation in patients with acute coronary syndromes: insights from the Rapamycin-Eluting Stent Evaluated At Rotterdam
Cardiology Hospital (RESEARCH) registry. J Am Coll Cardiol. 2003;41(11):2093-9.

9. Costa RA, Moussa ID. Percutaneous treatment of coronary bifurcation lesions in the era of drug-eluting stents. Minerva Cardioangiol. 2006;54(5):577-89.

10. Mauri L, Silbaugh TS, Wolf RE, Zelevinsky K, Lovett A, Zhou $Z$, et al. Long-term clinical outcomes after drug-eluting and bare-metal stenting in Massachusetts. Circulation. 2008;118(18): 1817-27.

11. Kirtane AJ, Gupta A, lyengar S, Moses JW, Leon MB, Applegate $R$, et al. Safety and efficacy of drug-eluting and bare metal stents: comprehensive meta-analysis of randomized trials and observational studies. Circulation. 2009;119(25):3198-206.

12. De Luca G, Stone GW, Suryapranata H, Laarman GJ, Menichelli $M$, Kaiser $C$, et al. Efficacy and safety of drug-eluting stents in ST-segment elevation myocardial infarction: a meta-analysis of randomized trials. Int J Cardiol. 2009;133(2):213-22.

13. Mauri L, Silbaugh TS, Garg P, Wolf RE, Zelevinsky K, Lovett A, et al. Drug-eluting or bare-metal stents for acute myocardial infarction. N Engl J Med. 2008;359(13):1330-42.

14. Montalescot G, Ongen Z, Guindy R, Sousa A, Lu SZ, Pahlajani $\mathrm{D}$, et al. Predictors of outcome in patients undergoing PCI: results of the RIVIERA study. Int J Cardiol. 2008;129(3): 379-87.

15. Yan BP, Clark DJ, Buxton B, Ajani AE, Smith JA, Duffy SJ, et al. Clinical characteristics and early mortality of patients undergoing coronary artery bypass grafting compared to percutaneous coronary intervention: insights from the Australasian Society of Cardiac and Thoracic Surgeons (ASCTS) and the Melbourne Interventional Group (MIG) Registries. Heart Lung Circ. 2009; 18(3):184-90.

16. Park DW, Park SW, Park KH, Lee BK, Kim YH, Lee CW, et al. Frequency of and risk factors for stent thrombosis after drugeluting stent implantation during long-term follow-up. Am J Cardiol. 2006;98(3):352-6.

17. Sousa A, Costa JR Jr, Moreira AC, Cano M, Maldonado G, Costa RA, et al. Long-term clinical outcomes of the DrugEluting Stents in the Real World (DESIRE) Registry. J Interv Cardiol. 2008;21(4):307-14.

18. Mattos LA, Lemos PA, Rassi Junior A, Marin-Neto JA, Sousa A, Devito FS, et al. Diretrizes da Sociedade Brasileira de Cardiologia - Intervenção Coronária Percutânea e Métodos Adjuntos Diagnósticos em Cardiologia Intervencionista (II Edição - 2008). Arq Bras Cardiol. 2008;91(6 Supl 1):1-58.

19. Grines CL, Bonow RO, Casey DE Jr, Gardner TJ, Lockhart PB, Moliterno DJ, et al. Prevention of premature discontinuation of dual antiplatelet therapy in patients with coronary artery stents: a science advisory from the American Heart Association, American College of Cardiology, Society for Cardiovascular Angiography and Interventions, American College of Surgeons, and American Dental Association, with representation from the American College of Physicians. Circulation. 2007;115(6):813-8.

20. Cutlip DE, Windecker S, Mehran R, Boam A, Cohen DJ, van Es GA, et al. Clinical end points in coronary stent trials: a case for standardized definitions. Circulation. 2007;115(17): 2344-51.

21. Daemen J, Wenaweser P, Tsuchida K, Abrecht L, Vaina S, Morger $\mathrm{C}$, et al. Early and late coronary stent thrombosis of sirolimus-eluting and paclitaxel-eluting stents in routine clinical practice: data from a large two-institutional cohort study. Lancet. 2007;369(9562):667-78.

22. Sianos G, Papafaklis MI, Daemen J, Vaina S, van Mieghem $\mathrm{CA}$, van Domburg RT, et al. Angiographic stent thrombosis after routine use of drug-eluting stents in ST-segment elevation myocardial infarction: the importance of thrombus burden. J Am Coll Cardiol. 2007;50(7):573-83. 
23. Feres F, Costa JR Jr, Abizaid A. Very late thrombosis after drugeluting stents. Catheter Cardiovasc Interv. 2006;68(1):83-8.

24. Virmani R, Farb A, Guagliumi G, Kolodgie FD. Drug-eluting stents: caution and concerns for long-term outcome. Coron Artery Dis. 2004;15(6):313-8.

25. Cook S, Wenaweser $P$, Togni M, Billinger M, Morger C, Seiler $\mathrm{C}$, et al. Incomplete stent apposition and very late stent thrombosis after drug-eluting stent implantation. Circulation. 2007; 115(18):2426-34.

26. Joner M, Finn AV, Farb A, Mont EK, Kolodgie FD, Ladich $\mathrm{E}$, et al. Pathology of drug-eluting stents in humans: delayed healing and late thrombotic risk. J Am Coll Cardiol. 2006; 48(1):193-202.

27. Fukuda D, Tanaka A, Shimada K, Nishida Y, Kawarabayashi T, Yoshikawa J. Predicting angiographic distal embolization following percutaneous coronary intervention in patients with acute myocardial infarction. Am J Cardiol. 2003;91(4):403-7.

28. Stone GW, Dixon SR, Grines CL, Cox DA, Webb JG, Brodie BR, et al. Predictors of infarct size after primary coronary angioplasty in acute myocardial infarction from pooled analysis from four contemporary trials. Am J Cardiol. 2007;100(9):1370-5.

29. Suenari K, Shiode N, Shirota K, Ishii H, Goto K, Sairaku A, et al. Predictors and long-term prognostic implications of angiographic slow/no-flow during percutaneous coronary intervention for acute myocardial infarction. Intern Med. 2008;47(10): 899-906.

30. Lincoff AM, Califf RM, Anderson KM, Weisman HF, Aguirre $\mathrm{FV}$, Kleiman NS, et al. Evidence for prevention of death and myocardial infarction with platelet membrane glycoprotein Ilb/IIla receptor blockade by abciximab (c7E3 Fab) among patients with unstable angina undergoing percutaneous coronary revascularization. EPIC Investigators. Evaluation of 7E3 in Preventing Ischemic Complications. J Am Coll Cardiol. 1997; 30(1):149-56

31. Costantini CO, Stone GW, Mehran R, Aymong E, Grines $\mathrm{CL}$, Cox DA, et al. Frequency, correlates, and clinical implications of myocardial perfusion after primary angioplasty and stenting, with and without glycoprotein Ilb/IIla inhibition, in acute myocardial infarction. J Am Coll Cardiol. 2004; 44(2):305-12
32. de Lemos JA, Antman EM, Gibson CM, McCabe $\mathrm{CH}$, Giugliano RP, Murphy SA, et al. Abciximab improves both epicardial flow and myocardial reperfusion in ST-elevation myocardial infarction. Observations from the TIMI 14 trial. Circulation. 2000;101(3):239-43.

33. Petronio AS, Rovai D, Musumeci G, Baglini R, Nardi C, Limbruno $U$, et al. Effects of abciximab on microvascular integrity and left ventricular functional recovery in patients with acute infarction treated by primary coronary angioplasty. Eur Heart J. 2003;24(1):67-76.

34. Lansky AJ, Costa RA, Mehran R, Mori K, Cristea E, Silva EC, et al. Implications of Myocardial Blush Grade (MBG) on late clinical outcomes after mechanical reperfusion in Acute Myocardial Infarction (AMI) and its interaction with different biomarkers of reperfusion: results from the EMERALD trial. Circulation. 2005;112(17):II-766.

35. Spaulding C, Henry P, Teiger E, Beatt K, Bramucci E, Carrie $D$, et al. Sirolimus-eluting versus uncoated stents in acute myocardial infarction. N Engl J Med. 2006;355(11):1093-104.

36. Stone GW, Lansky AJ, Pocock SJ, Gersh BJ, Dangas G, Wong SC, et al. Paclitaxel-eluting stents versus bare-metal stents in acute myocardial infarction. N Engl J Med. 2009;360(19): 1946-59.

37. Lemos PA, Saia F, Hofma SH, Daemen J, Ong AT, Arampatzis $\mathrm{CA}$, et al. Short- and long-term clinical benefit of sirolimuseluting stents compared to conventional bare stents for patients with acute myocardial infarction. J Am Coll Cardiol. 2004; 43(4):704-8.

38. Slottow TL, Steinberg DH, Roy P, Javaid A, Buch AN, Okabe $\mathrm{T}$, et al. Drug-eluting stents are associated with similar cardiovascular outcomes when compared to bare metal stents in the setting of acute myocardial infarction. Cardiovasc Revasc Med. 2008;9(1):24-8.

39. Chen MS, John JM, Chew DP, Lee DS, Ellis SG, Bhatt DL. Bare metal stent restenosis is not a benign clinical entity. Am Heart J. 2006;151(6):1260-4.

40. Doyle B, Rihal CS, O'Sullivan CJ, Lennon RJ, Wiste HJ, Bell $\mathrm{M}$, et al. Outcomes of stent thrombosis and restenosis during extended follow-up of patients treated with bare-metal coronary stents. Circulation. 2007;116(21):2391-8. 